that no excess runs down the throat. Lastly, I would strongly urge that all those who use instruments of any sort within the mouth should make it an invariable rule to sterilise them both before and after use.

\section{ILLUSTRATIVE CASES OF GUNSHOT WOUNDS OF THE SKULL AND BRAIN. ${ }^{1}$}

BY L. G. IRVINE, M.A., M.D., B. Sc. Edin., LATELY CIVIL SURGEON, SOUTH AFRICAN FIELD FORCE.

THE following notes are based on the observation of 30 cases of gunshot wounds of the skull and brain which came under my own care or that of several of my colleagues in the military hospitals in South Africa during the war and on which I made direct personal observations. My thanks are due to those of my colleagues who kindly allowed me to make use of their cases for this purpose.

The cases which I have chosen for description have been selected with the sole object of illustrating the various types of injury to the skull and brain caused by the Mauser or LeeMetford bullet and I have in order to make this paper as brief as possible taken one case only of each type. I would not, however, have you as a consequence conclude that $I$ am in any way arguing from single instances, since the material from which these cases are selected, if not very extensive, is at all events considerable and representative. Further, $I$ have included in the series several fatal cases. I have done so because in them post-mortem examination allowed of a more exact observation. Had my object been merely to describe successful cases of brain surgery a very different selection might have been made.

Gunshot injuries of the skull and brain still, as formerly, remain the most fatal wounds of all and still exact their heavy toll of deaths upon the field. Injuries of this sort are naturally most common in engagements fought at close range, especially where the opposing troops are more or less under cover or are actually entrenched. I hope that in time some surgeon who has had extensive experience in the field will be able to contribute to the surgical literature of the war a study of the nature of the wounds which kill. The study would be a valuable one, for it is rarely possible to subject the dead on the battle-field to exact examination; circumstances do not usually permit of it-the wounded and not the dead claim the first attention of the surgeon. But $I$ think one may safely say that of all those who are killed outright upon the field, in certainly the majority death is due to wounds of the skull and brain. One surgeon, who had had exceptional experience of field work, stated to me his belief that penetrating wounds of the skull and brain accounted for 60 per cent. of those killed in action. Of the remainder the majority were shot through the chest, while in a further proportion death was due to penetrating wounds of the abdomen. Only a comparatively small percentage of patients died on the field from primary external hæmorrhage. If this be so it is obvious that, of actual wounds of the skull and brain, the proportion which are immediately or rapidly fatal must be a very high one, especially when we take account of the further fact that a considerable number of those who are carried from the field alive die in the field hospitals within a few hours or days thereafter. Perhaps it is not an exaggeration to say that of all penetrating gunshot wounds of the skull seven out of 10 are immediately or rapidly fatal, while of those patients who are taken from the field alive probably not more than half actually recover. Nevertheless, the actual number of recoveries from injuries of this sort has been sur. prising and has certainly been very much higher during the war in South Africa than in any previous one. This has been due in part, of course, to the more systematic use of antiseptics, but also, and in a much greater degree, to the properties of the small-bore bullet itself, its small size, its high penetrative power, its smaller liability to distortion, and its general initial asepticity.

Otis records that in the American Civil War in only 14 cases of complete perforation of the skull by rifle bullets

${ }^{1}$ A paper read before the Transvaal Medical Society. were the patients subsequently discharged as pensioners and that "the patients who did survive were quite incapacitated from earning a livelihood by physical or mental exertion."

Now, fatal as gunshot wounds of the skull and brain have been during the war in South Africa, there is no doubt that a considerable number of patients (perhaps 15 per cent.) have survived these injuries. Almost every military surgeon must have seen several. I myself have made observations on at least 12 cases in which recovery took place after complete perforation of the cranial cavity. It is interesting therefore to inquire in what class of cases this result has been attained.

Gunshot Injuries at Close Range.

The factors determining the nature and gravity of gunshot injuries of the skull and brain are mainly two: (1) the range at which the wound is inflicted; and (2) the region of the skull and brain involved, gunshot fractures of the base being of course much more grave than fractures of the vault. Each of these factors has its importance. The first two cases which I shall cite are examples of gunshot wounds of the skull at a very close range. Cases of this sort do not often occur in action; of the instances which I shall cite one was a case of suicide and the other of accidental death.

CASE 1.-This case was ghastly enough. An artilleryman prompted to suicide by drink and the monotony of the war, shot himself through the head with his carbine. The muzzle of the carbine-the ordinary service weapon with a 303 bullet-was against, or in very close proximity to, his head and therefore the direct effect of the explosion of the cordite was superadded to the effect of the bullet. Practically the whole of the vault of the skull was broken up into six or seven large loose fragments, roughly held together by the scalp, and the scalp itself was ripped completely across from the entrance wound in the right anterior temporal region to the exit wound in the left parietal area. From the exit wound a considerable area of bone had been carried out. The vault, indeed, was literally "blown to pieces," although the base of the skull, beyond some fissuring, was practically intact. The brain was utterly destroyed.

CASE 2. - In contrast with this was a second case, that of a girl who was accidentally killed by the bullet from a LeeMetford rifle, discharged at a distance of three paces. The difference was very striking, the injuries in this case being much less extreme than one might have expected. The following notes of the post-mortem examination were furnished to me by Dr. R. P. Mackenzie, district surgeon, Johannesburg. "The entrance wound in the right malar bone showed no sign of fissuring, the bullet having drilled a clean small hole. The skin showed no sign of singeing. The exit wound in the occipital bone, a little to the right of the protuberance, was larger, freely admitting the forefinger, and there was a zone of stellate fissuring round this, but this fissuring did not run far into the vault and there were no loose fragments. Some bone débris was found in the track of the bullet through the brain, which was rather more than an inch in diameter. The scalp wounds corresponded in size to those in the bone. Death, of ccurse, was instantaneous." It is obvious that here, although the local injury was great, the general integrity of the skull and the scalp was preserved.

These two cases, it seems to me, are useful as standards and are of value medico-legally. In Case 1 the weapon was discharged in contact with the head and in Case 2 at the short distance of three paces. The size of the bullet and the explosive charge were absolutely alike, the weapons being very nearly identical. The striking difference between the extreme degree of destruction manifested in the one and the comparatively localised character of the injuries in the other must therefore have been due to the fact that while in the first the direct explosive effect of the cordite was superadded to the mechanical effect of the bullet in the second the effect of the bullet alone was present.

There has been a good deal of loose talk during the war of heads that have been blown to pieces and of explosive bullets and the like. Hence I think that these cases form a useful basis for discussion. I do not believe that the Mauser or Lee-Metford bullet by itself, even at very close range, will "blow the top of the head off." At the same time I grant that the injury may be greater than in the case of the girl which I have cited. In that case the base and vault were both involved. In wounds of the vault alone the fissuring may be much greater and may connect the apertures of entrance and exit since the wounds of entrance and exit are R 2 
here closer together and the bullet track subtends a continuous arc of bone. The nearer they are together the greater the likelihood of fissuring extending between them. Fissuring, of course, is almost always greater round the exit wound. I show a specimen of this type (Fig. 1) which usefully supplements Case 2. The injury was certainly inflicted at a greater range, but it seems to me probably to exemplify what must be nearly the extreme degree of injury to the skull produced by a Mauser or a Lee-Metford bullet. The subject of it lived for 12 days.

Such injuries show, in short, the typical so-called "explosive effects" caused by the solid small-bore bullet at close range, with the characters of small entrance and large exit wounds and great destruction of the soft tissues. Similar and even more exaggerated effects are shown in close-range gunshot wounds of the shafts of the long bones, which have during the war been frequently, and in many cases erroneously, attributed to the use of "expanding" bullets. I have seen in the back of the thigh an exit wound of three by four inches produced in a gunshot points about such " expanding" bullets are their lower penetrative power and greater liability to distortion. Range for range, therefore, one would certainly expect the injuries produced by them to be the more destructive, but that the normal small-bore bullet at very close ranges produces injuries of an "explosive" type is quite undoubted, and, on the other hand, as I shall show later, an "expanding" bullet may penetrate the skull without expanding.

\section{Gunshot InJURIES at MEdIUM RANGes.}

I think that I have now said enough in illustration of the characters of penetrating gunshot wounds of the head at a very close range. Whether they involve the base or the vault they are, if the cranial cavity be penetrated, uniformly fatal. Indeed, I think one may extend this statement so far as concerns gunshot fractures of the base and say that all gunshot fractures of the base involving the middle or posterior fossa or both, with penetration of the cranial cavity and injury to the base of the brain, are at all ranges either immediately "or ultimately fatal. They

FIG. 1.

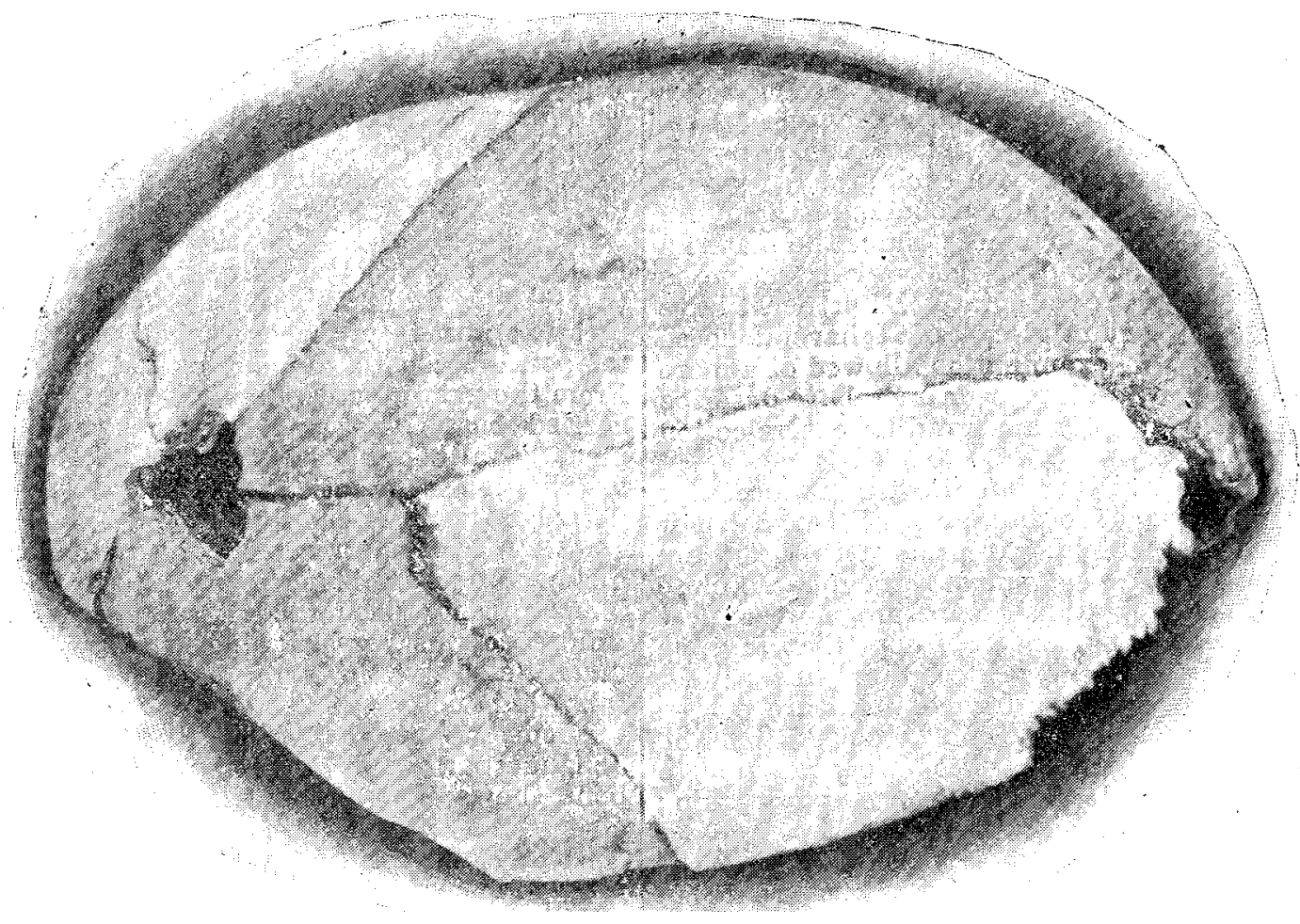

Close-range gunshot fracture of the vault, produced by a small-bore bullet. The anterior aperture, an inch above the anterior inferior angle of the left parietal bone, has been considerably enlarged by the gouge forceps. The specimen shows especially the infence fissuring with the posterior wound; the latter is at the posterior superior angle of the left parietal bone.

fracture of the femur by a solid Lee-Metford bullet at a range of 50 yards where there was no question at all of the use of an "expanding" bullet. Not only so, but it is certain also that the normal Mauser or Lee-Metford bullet will, at a close range, itself become distorted on impact with such a bone as the femur, tibia, or humerus, and so become in effect an expanding bullet. The bones of the skull, however, are not sufficiently resistant to produce this distortion in a normal bullet. My belief therefore is that where the direct explosive effect of the cordite or melinite is absent the effect of the LeeMetford or Mauser bullet, even at ranges of from five to 50 yards, is not greater than in the case of the girl which $I$ have described or of the specimen which I have shown. The local destruction is great and fissuring may be extensive but the general structural integrity of scalp and cranium is preserved. The head is not "blown to bits." Consequently, where effects greater than those which I have indicated are present they must, I think, be attributed to the use of bullets of a larger calibre than the Lee-Metford or Mauser, or of bullets of an illegitimate character, such as the Jeffrey's sporting or the soft-nosed bullet, which have undoubtedly been used in a certain proportion of cases during the war. In many instances, however, it must be difficult, if not impossible, to draw the line. The main differentiating form the commonest type of injury among those who die from wounds of the head on the field of battle; many are hit about the orbits or the root of the nose, the bullet passing out through the occiput. If there are any exceptions to this rule they must be extremely few. Where the anterior fossa alone is involved the injury is of course less serious. I need not therefore detain "you by the description of any cases of this type; those which I have seen have been invariably fatal.

As the range at which the wound is inflicted increases the character of the injury becomes progressively less severe. As a consequence medium-range penetrating gunshot fractures of the vault are not necessarily fatal; a certain proportion of the patients indeed ultimately recover. A considerable number of these cases came under my own observation, and in these the wounds were almost all received at ranges of from 500 to 800 yards. Some may have been at rather shorter distances, but the estimates of range given by patients who have sustained such severe wounds of the head are clearly especially liable to error. These injuries fall into two main classes, those causing depressed or gutter fractures, and those causing complete perforation of the cranial cavity with separate entrance and exit wounds in the skull. If the line of impact of the bullet on the vault of the skull is tangential 
a depressed or gutter fracture is produced; if, on the other hand, the line of impact is more perpendicular a complete perforation of the cranial cavity results, the entrance and exit wounds in the bone being more or less widely separated. It sometimes happens, of course, that there is penetration of the skull without complete perforation, the bullet not making its exit but remaining lodged in the cranial cavity. It is sufficient in the meantime merely to mention this fact. It may happen also that the bullet striking a glancing blow on the skull may cause symptoms of concussion or of temporary paralysis without any obvious injury to, or depression of, the bone, although it is, of course, possible that in some instances of this nature there may be fracture of the inner table without obvious depression of the outer. But of the actual gunshot fractures of the vault which we see in the military hospitals the main types are those which $I$ have mentioned and these are quite distinct in character, symptoms, and prognosis.

\section{Depressed and GutTer Fractures.}

Of depressed and gutter fractures those of the latter type, the gutter or trough fracture, are the more common. I shall therefore confine my description to them. In such cases there are two distinct wouncls in the scalp with a single gutter or trough fracture through the skull. At close ranges the local destruction so produced may be very considerable; the trough may be from half an inch to an inch wide, with fissuring extending from its extremities, and splinters of bone may be driven deeply into the lacerated brain substance. 1 have notes of one such case at a range of under 100 yards.

FIG. 2.

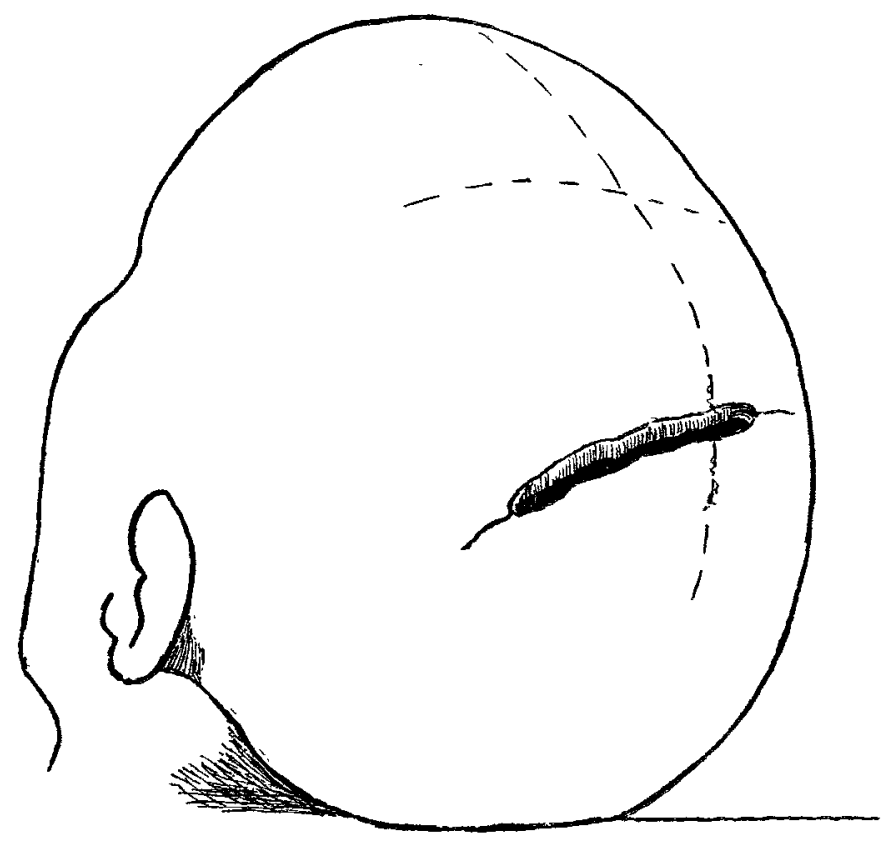

FIG. 2 A.

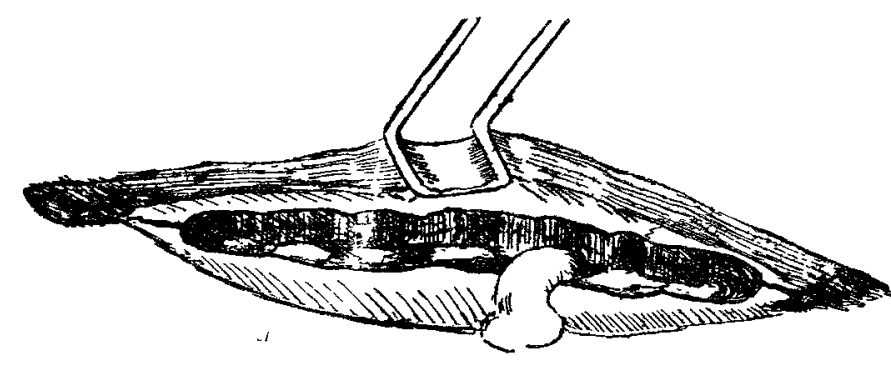

Gutter or trough fracture of the vanlt caused by a Mauser Fig. 2 A the details of the gutter or trough in the skull.

In wounds of this type, however, sustained at a medium range, the injury, in my experience, is much less severe. I show a sketch of a case of this nature (Case 3, Figs. 2 and 2 A) the appearances of which are very characteristic of such injuries at a medium range. In four cases which I saw the trough was from one-third to half an inch in width with sharp-cut edges and averaged from two and $a$ half to three inches in length. The bone represented by it was comminuted through both the outer and inner tables, small loose fragments of both tables forming the floor of the trough and being driven sometimes underneath the sound bone towards the exit wound. Only one case showed any fissuring of the skull and in it there was merely a single fine fissure running a short distance from either end of the troughindeed, the limitation of the injury to the bone was in these four cases quite remarkable. The dura mater was lacerated in all and to a varying extent the cerebral cortex also. Three of the four cases presented no special symptoms except that cerebration was slow in one, but in the last, the one which I have illustrated, there was marked cerebral irritability with twitching, followed by paralysis of the right arm, hand, and leg, due to laceration of the brain and hæmorrhage from the superior longitudinal sinus. All four cases were operated on by exploration and enlargement of the gutter or trough by the gouge forceps. All the patients recovered, and not only so, but all but the last seemed on dismissal in a fair way to regain their normal mental power. Too short a time, however, had elapsed for me to speak at all definitely regarding the likelihood of this improvement being progressive or permanent.

\section{Supereictal Perforating Fractures.}

Suppose, now, that the line of impact of the bullet strikes the skull more fairly. In this case two separate apertures of entrance and exit are produced in the bone in place of the single gutter or trough caused by the tangential blow. These injuries may therefore be called "perforating fractures."

Now, clinical observation shows that alike in regard to physical characters, clinical symptoms, and prognosis, these perforating fractures may be divided into two relatively distinct types which I may call the superficial and the deep perforating fractures. In the superficial perforating fracture the course of the bullet more nearly approaches the tangential ; it runs close under the skull, subtending a shallow arc of bone. Such a line is shown in the sketch (Fig. 3) as

FIG. 3.

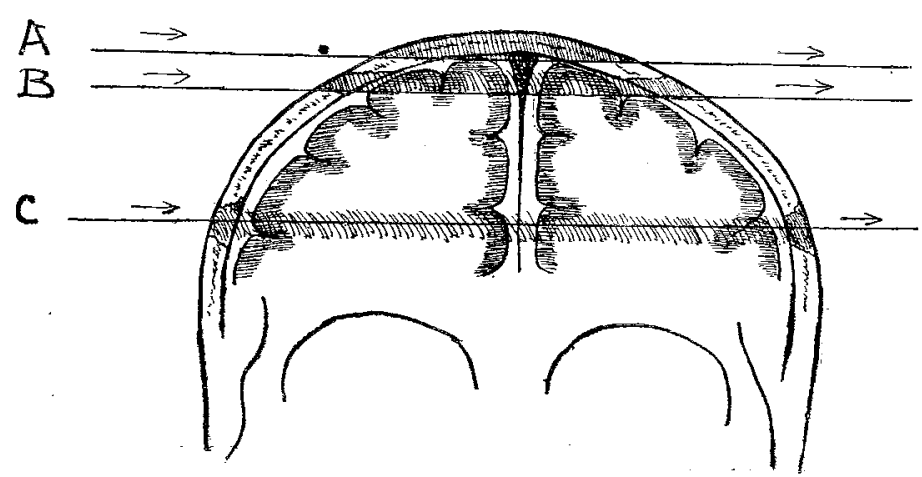

Diagram showing the modes of production of the main types of gunshot fracture of the vault. $A$, Gutter or trough of gunshot fracture of thef pault. 1 , Gutter or trough ever, usually occur in the long axis of the skull. C, Deep perforating fracture.

B, A being the tangential line of the gutter fracture. Now cases of this nature at a medium range present a marked similarity of type and the injury is much more severe than that of the deep perforating fracture which $\mathbf{I}$ shall presently describe.

In the superficial perforating type the bullet strikes the skull obliquely, causing an oval or irregular wound of entrance and a similar or somewhat larger wound of exit which may be half an inch or more in diameter. Travelling as it does close under the skull it produces fissuring of the bone, the fissure being often single and connecting the apertures of entrance and exit. At shorter ranges, indeed, these fractures approximate to the more severe type of injury shown in Fig. 1. A lacerated track is produced in the surface of the brain, the cortex is ploughed through, and the grey matter is liable to extensive injury. Cerebral irritability is a marked symptom. Fractures of this type are naturally especially liable to occur when the line of impact is more or less antero-posterior, in the long axis of the skull, 
in which direction the cranial curvature is flatter than in the transverse diameter, but they occur also in cases where the course of the bullet is more or less transverse, provided that the arc of the vault subtended by it is a small one. Most of those cases which I saw, however, were, as I have said, more or less in the long axis of the skull. The superior longitudinal sinus is specially liable to injury in wounds of this type of the vertex in the middle line and in these cases more or less complete hemiplegia is common. All the cases were subjected to operation either by trephining or by enlargement of the apertures in the skull. In one case a hernia cerebri developed after operation. I mention this merely to refer to the satisfactory local results obtained by painting the surface of the tumour with a 20 per cent. solution of formalin and shaving off the resulting crust every three or four days. So far, however, as I have seen, and taking into account the immediate prognosis only, the majority of these cases in which the injury has been inflicted at a medium range and in which the patients live to reach the stationary and general hospitals, recover ; in what proportion of the total number of wounds of this type recovery ensues is a question that I cannot so definitely answer.

All of the above features are illustrated in the sketch (Fig. 4) which I show here. It was made at the time of

FIG. 4.

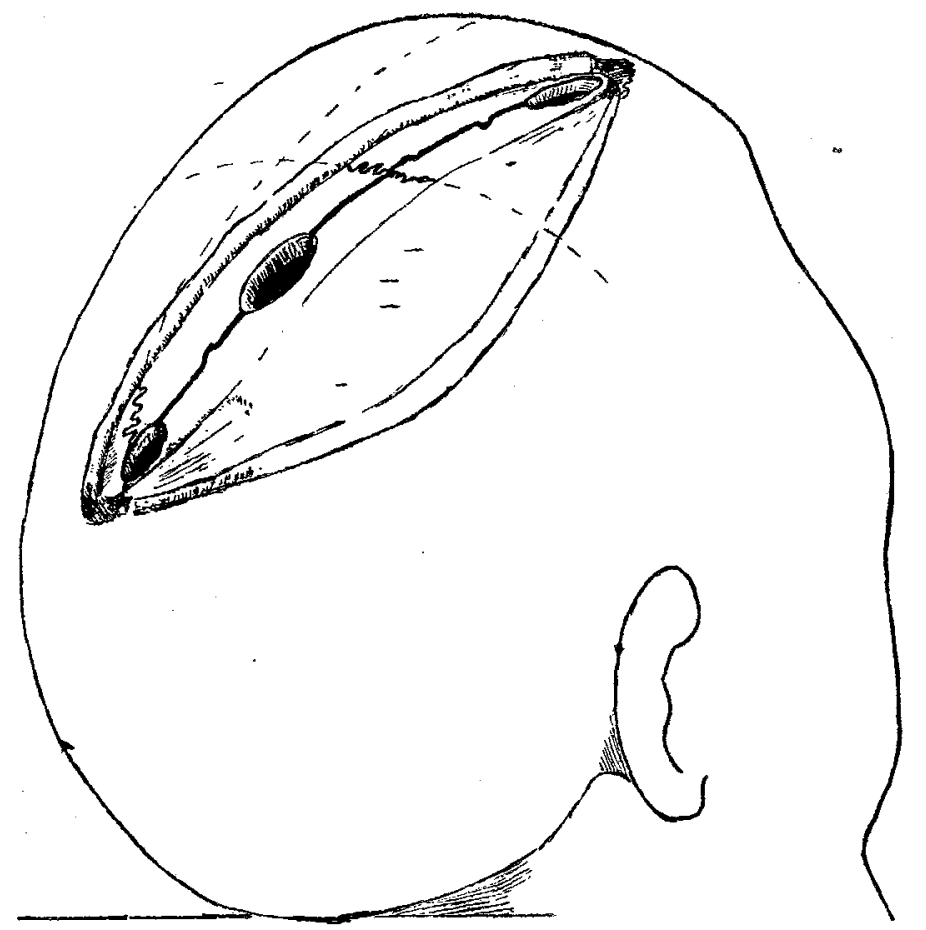

Superficial perforating fracture of the vault as shown at the time of operation. Note the trephine opening and the single fissure connecting the apertures of entrance and exit. The lines of the coronal and sagittal sutures are also indicated.

operation from the case of a young officer wounded at Colenso (Case 4). Note that the wound is in the long axis of the skull. Note the small oval entrance and exit wounds, the former on the vertex in the middle line and the latter in the upper frontal region one and a half inches to the right of the middle line. Note also the single fissure connecting them. In this case the superior longitudinal sinus was wounded and there were complete paralysis of the left arm and partial paralysis of the left leg and face. He was semi-conscious when operated on 36 hours after receipt of the injury and there was free venous oozing from the anterior wound and from the line of the fissure with escape of disorganised brain matter from the former. The anterior wound was enlarged and plugged with gauze and a circle of bone was then removed midway between this and the posterior wound at a point where the bleeding seemed to be most profuse. The dura mater, which was bulging and not pulsating, was opened. This wound was then also plugged. The patient made a good recovery. A month afterwards the power of the arm was almost normal and there was good recovery of the power of movement of the knee and hip but foot-drop persisted. Cerebration was fair though a little slow, but the patient could read a novel through with enjoyment. It is scarcely possible, however, that recovery will ever be complete enough to enable him to resume any active work. The case was under the care of Dr. D. W. Johnston.

\section{Dege Perforating Fractures.}

Let us now take the type of case which I have called the "deep perforating fracture." The actual distinction between this and the previous type lies in the fact that the course of the bullet is in this case deeper and subtends a larger arc of the skull. It is indicated by the line $\mathrm{C}$ in Fig. 3 . The injury to the bone and to the cortex of the brain is thereby much lessened. This character serves to differentiate it, both in physical and clinical features and prognosis, from the former type. Associated with this is the further fact that in these injuries the track of the bullet is usually more or less in the transverse axis of the skull, in which direction, of course, the cranial curvature is fuller and less shallow. I saw nine cases of this type and in all but one the track of the bullet crossed the midale line ; in four of these, indeed, the bullet passed more or less directly transversely through both parietal bones.

Now here again the cases were all with minor variations of one general type. The bullet, striking the skull at, or nearly at, right angles, drills it and, traversing the substance of the brain more deeply than in the case of the superficial perforating fracture, drills the skull again at the point of exit. The apertures in the bone are small and, as a rule, similar. Frequently they are of the circular type shown in the specimen, taken from one of the cases in which the bullet passed almost directly transversely through both parietal bones. Note the exit wound (Figs. 5 and $5 \mathrm{~A}$ ), a clean drill with a ring-like bevelling off of the outer table. An exactly similar condition was seen at the entrance wound, although the symmetry of this was spoilt by the trephine and $I$ have therefore not reproduced it. It also showed a clean drill with a corresponding bevelling of the inner table. The direct injury to the bone is here really at a minimum. There is, indeed, barely space to let the bullet through. Sometimes, it is true, the apertures are larger and irregular or roughly rectangular and if this is so the exit wound is usually the larger of the two and when loose spicules of bone are removed it may have a diameter of three-quarters of an inch or more.

Another noteworthy feature is the absence of fissuring in most cases of this type. In the majority of those which I examined there was no fissuring of the bone whatever and in the instances in which it did occur it was slight, a single fine fissure only running for a short distance from the exit wound. This condition is in decided contrast to the marked fissuring which characterised the cases of superficial perforating fracture. In one of the two cases examined post mortem a minute spicule of bone was found at some depth in the brain substance, but apparently such fragments are not carried in deeply to any great extent. They lie on the dura mater or superficially in the brain substance close to the entrance wound. Laceration and disintegration of the brain substance along the direct track of the bullet are in thesa cases considerable-more so than one would expect from the character of the wounds in the skull. In most cases disorganised brain matter exuded from the wound in the bone and sometimes in considerable quantity. In one case the longitudinal sinus was wounded; in two cases the middle meningeal artery ; in no case did these complications ultimately prejudice the result. In one case the bullet had just failed completely to perforate the skull, and was found on exploration neatly impacted in the bone at the exit wound, the anterior half of the bullet projecting from the skull. It was removed with some difficulty by the lion forceps.

The symptoms prior to operation are typically those of laceration of the brain. The classical state of mental or cerebral irritation characteristic of this condition is usually well marked and the temperature and pulse are raised unless compression is markedly present. There may be in addition localising symptoms, twitching, localised paralysis or hemiplegia, or even, in the gravest cases, general convulsions. But paralytic symptoms are perhaps less common, and certainly less extensive, in injuries of this nature than in superficial perforating fractures and the general character of the wounds is decidedly less grave. Of the nine cases eight were operated on. In two the entrance wound was alone explored, in three the exit wound, and in three both wounds. In the absence of localising symptoms pointing to the exit 
wound or to some other area it is always wise first to explore the aperture of entrance in the skull, since it is from it that spicules will be carried inwards into the dura mater and the brain substance. If the condition of the patient allows of it both wounds should be explored. It is not usually necessary to trephine; loose spicules should be removed and the open-

FIG 5.

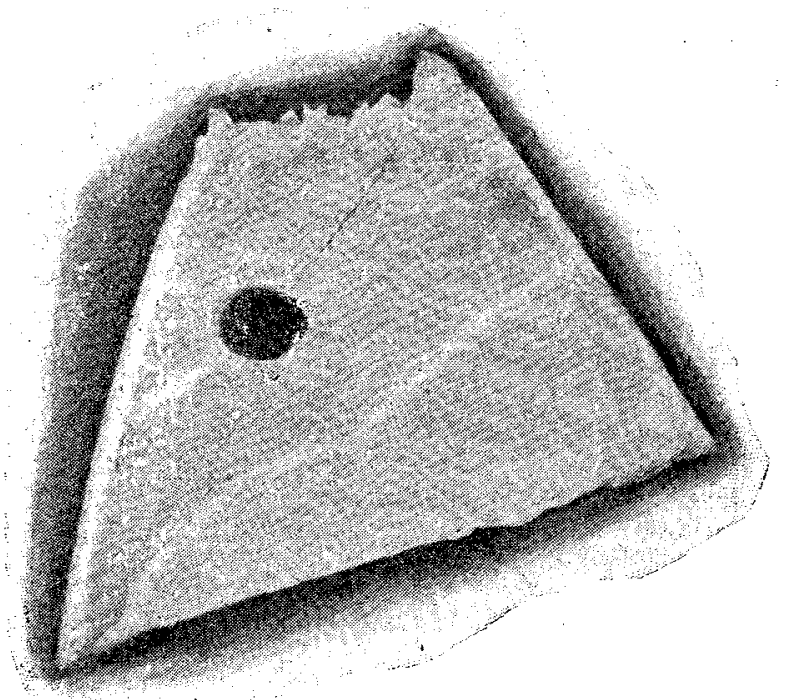

FIG. 5 A.

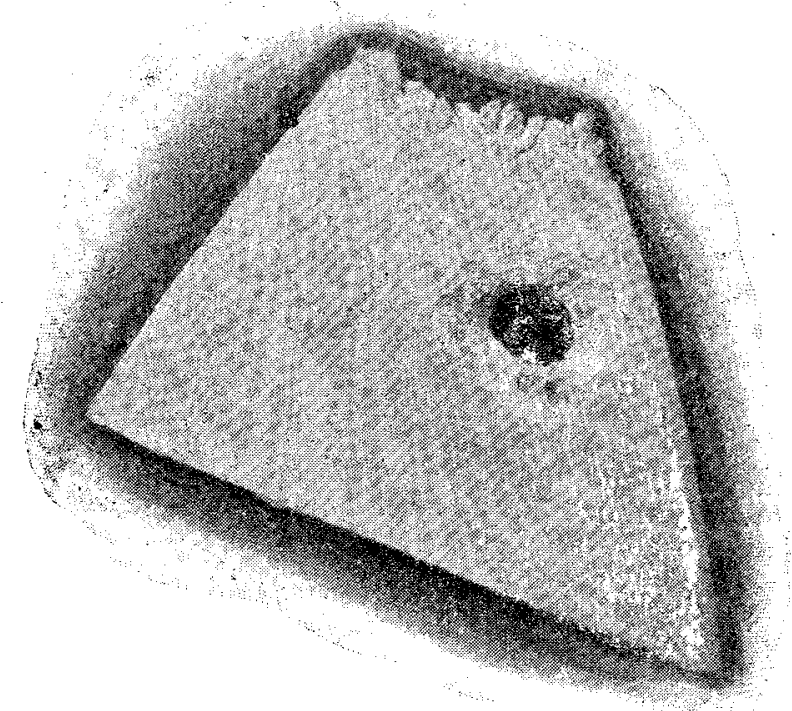

Medium-range deep perforating fracture of the vault by a Mauser bullet which passed almost directly transversely through both parietal bones. The aperture of exit alone is shown, but that of entrance quite corresponded. Fig. 5 is a view of the inner surface of the skull at the aperture of exit and shows a clean circular drilling of the bone with a single fine fissure running from it. The actual diameter of the aperture was less $t$ han $\frac{1}{3}$ rch of an inch ( $\frac{18}{6} 8$ ths of an inch). Fig. $5 \mathrm{~A}$ shows the external surface, the

ing enlarged where necessary by Horsley's gouge forceps which I have found very useful. I shall only cite one case in illustration of this type of injury.

CASE 5.-A private was wounded on Feb. 23rd, 1900, in the first assault on the Pieter's position, the range being rather under 500 yards. The entrance wound was just below and to the right of the external occipital protuberance and the exit wound was one and a quarter inches above and behind the left external auditory meatus. The wounds in the scalp were those of a normal Mauser bullet and were quite similar. On admission to hospital two days later he was stupid but conscious; he was excessively irritable; there was no paralysis; the pupils were dilated but reacted sluggishly; the temperature was $101^{\circ} \mathrm{F}$. On the 27th the exit wound was explored; loose spicules of bone were found on the dura mater and when these were removed the resulting wound was roughly rectangular, measuring three-quarters of an inch by one and a half inches. Nearly an ounce of disorganised brain tissue and blood was exnded, there being clearly considerable intracranial pressure. The brain then pulsated. There was a single fine fissure in the skull running backward. The entrance wound was not explored. On the next day the symptoms of cerebral irritation had passed off and two days later the patient was doing well and was sensible. The temperature was normal after the operation. An interesting symptom was present in this case which was due to the injury to the left occipital lobe or its connexion with the left temporosphenoidal lobe-namely, amnesia verborum. Speech was slow but well coördinated, but he could not recollect the names of the simplest articles when shown to him, although when the proper names were verbally suggested he could both articulate and write them. $\mathrm{He}$ also had dimness of vision, especially in the left eye. Both of these symptoms gradually passed off and by the end of March the amnesia had nearly disappeared and he was then quite intelligent.

of these nine cases of deep perforating fracture death occurred in two, both I think being due to septic encephalitis; both had been subjected to operation; seven patients recovered. Thus the immediate prognosis of the majority of these cases where the patients live to reach the stationary or general hospitals is good. It is possible, indeed, that an actual majority of all cases of medium-range deep perforating fractures of the vault recover. Indeed, one may assert with some approach to certainty that, while, as I have stated, penetrating gunshot fractures of the vault or the base at a close range are always fatal and from gunshot fractures of the base even at longer ranges recoveries must be extremely few, yet in medium-range fractures of the vault the immediate prognosis of gutter fractures is good and the prognosis of what I may call deep perforating fractures is decidedly better than that of the more superficial perforating fractures.

The ultimate prognosis is a different matter which only time can decide. It would be an interesting investigation for the proper authorities carefully to follow up the subsequent history of such cases as I have mentioned. The time at my disposal for observation was not sufficient to form a competent judgment on the matter, as those patients who recovered were invalided home, as a rule, about a month or six weeks after the receipt of the injury. Yet even within this short limit of time the progress made was in some cases surprising. I have already stated that I believe that three of the four cases of gutter fracture will apparently regain normal mental power, although in this estimate I may be unduly optimistic. Of the recoveries from superficial perforating fractures few, if any, would do so.

The six cases of recovery from deep perforating fractures which I was able to follow up were, I think, intermediate in character. In the first case, cerebration was fair but there was loss of vision in the left eye ; in the second, in which the patient had had paralysis of the muscles of the left shoulder and leg, there was good recovery of power in the former and the power over the leg was steadily improving; he was intelligent and read the newspaper with integest but soon became tired. The mental condition of the patient in the third case was the same and that of the patient in the fourth case, the subject of the amnesia which I have described, was similar. So also with the two other patients, one of whom had had partial motor aphasia and paresis of the right arm. All of these observations were taken a month after the receipt of the injury. I may say broadly that all the six patients could then talk quite intelligently about their wounds and other experiences but that their power of continued mental exertion as shown in reading or conversation was soon exhausted, in some more markedly than in others. Whether any of them would completely and permanently recover it is impossible for me to say. Several at least seemed in a fair way to do so. I trust, however, that cases of this nature will be carefully followed up by those whose position enables them to do so and the result published.

\section{Cases in which the Bullet lodged in the Cranial} CA ritry.

Let me now, in conclusion, quote one case more of another type, one in which the bullet penetrated the clanial carity aud remained lodged withn it. Stich injuries an lov 
ancommon; they occur, of course, at long ranges or from cicochets. They are usually fatal but not always so. The case has an additional interest from the fact that the injury was inflicted by a soft-nosed Mauser bullet.

CASE 6.-The patient, a private, was wounded in a minor engagement near Vereeniging and on the evening of the third day was admitted to the military hospital at Flandsfontein. A small circular wound of entrance could be seen over the prominence of the right malar bone, below and in a line with the outer canthus. No exit wound could be found and the bullet had clearly lodged in the cranial cavity. There was complete paralysis of the right arm and leg but no paresis of the face. The pupils were equal and reacted sluggishly to light. The patient was semi-conscious, presenting signs of advancing compression. On the next morning trephining was performed over the middle of the left motor area. The dura mater bulged and did not pulsate; it was therefore incised. The brain protruded somewhat. After the escape of a considerable quantity of blood pulsation returned. Search was then made with the probe for the bullet but it was not located. The patient died on the following morning early on the fifth day after receipt of the wound.

A post-mortem examination was made to determine the coarse and location of the bullet. The probe could be readily passed through a channel leading from the entrance wound through the right malar bone and the body of the sphenoid bone. Crossing the middle line the bullet track entered the cranial cavity in the middle fossa. There was no gross fissuring of the base and the channel through the bone was of small diameter. The bullet had then apparently impinged against the anterior surface of the petrous portion of the left temporal bone about its middle, at which point a small portion of lead was found. It had then been deflected directly upwards towards the vertex, producing a lacerated track in the brain substance. This entered the under surface of the anterior portion of the left temporo-sphenoidal lobe, not, however, appearing on the surface of the brain till it emerged at the vertex one and a half inches from the midale line. At this point a small puncture in the dura mater showed that the bullet had impinged against the skull.

had then been again deflected backwards and downwards through the substance of the ascending frontal and parietal convolutions. It was found lying at the middle of the latter very close to the operation wound. The ascending track was about an inch in diameter at its lower end ; the descending track was smaller.

This case presents several features of interest. It was caused by a soft-nosed Mauser bullet, one, that is, in which the point of the nickel sheath had been removed to the extent of a quarter of an inch, leaving the tip of the leaden core exposed and projecting. Such a bullet is of the "expanding" type, of which a good deal has been heard during the war. Nevertheless, it did not "expand," although it had drilled the malar and sphenoid bones and impinged against the dense substance of the petrous bone. The only distortion which it suffered was that the projecting leaden tip had been completely shorn off transversely at the point where the nickel sheath erded, presenting an appearance quite different from slue olique shear of a ricochet. As a consequence the venecrative power of the bullet was so reduced that it did not pass out of the cranial cavity, but being twice deflected by contact with the bone and altered in shape produced a very extensive laceration of the brain tissue which was the cause of death. Had the bullet been a normal Mauser it would at a medium range have completely perforated the cranial cavity; the injury to the bone would have been more grave but the laceration of the brain would have been less extensive. The question of exploratory operation which arines in such cases must depend for decision on the indicridual circumstances of each case. Should, as in this instance, localising symptoms render it probable that the bullet is lying in a situation accessible to the surgeon its removal should certainly be attempted.

Johannestorg.

New Operation Theatres at Bradford Royal TNFIRMARY. - Two new operation theatres provided at the Bradford Royal Infirmary by the generosity of Mr. Harrison Benn of Holcombe Hall, Dawlish, South Devon, were opened on Oct. 10th by Viscount Mountgarret. The instruments and furnishings were the gift of. Mr. W. H. Longbottom, of Myddleton Hall, Ilkley.

\section{MENTAL DISSOLUTION THE RESULT OF ALCOHOL. ${ }^{1}$}

By ROBER I JONES, M.D., B.S. LoND., F.R.C.S. ENG., PHYSICIAN ANI SUPERINTENDENT OF THE LONDON COUNTY ASYLUM, CEAYBURY.

WHEN, at the request of its able President and my old friend Dr. Harry Campbell, I consented to read a paper before the Society for the Study of Inebriety I felt the limitation of my experience in regard to the subject of inebriety, as ordinary cases of intemperance do not come under my care. My knowledge is derived from those in whom the vice of intemperance has become a disease, manifested by a craving over which a normal will has no control and resulting in acts which do not recognise submission to authority and which are judged to be irresponsible. The persons to whom I refer form only a small part of the multitude who indulge in strong drink, but they are by no means a small proportion of those who are admitted into asylums and who thereby sacrifice two objects so dear to self-respecting men-viz., their liberty and their legal franchise.

Many distinguished writers and persons of much authority and ability, too numerous to mention, have expressed themselves before your society upon this great question of drink, and its philosophical, medical, social, and economic aspects have been presented and discussed, but, not recently at any rate, have the effects of alcohol in relation to the production of insanity been submitted to its notice, for I have observed no such record in its proceedings of late years. If my paper fails to be of interest I trust that the President of the society may not be altogether exempt from the rebuke which in other circumstances would have been mine.

\section{The Distribution of Alcohol.}

Of all poisons, possibly alcohol is the most universal and the most widely diffused in nature, for it is found wherever destruction of organic matter by the various agents of fermentation takes place; and it is almost impossible to avoid it. We breathe it in the air, we drink it at the bubbling spring, and eren when we die we are laid in alcoholic earth. Like many other poisons it is from its stimulating properties of distinct service to mankind, and although its physiological action has given rise to much acrimonious debate the consensus of opinion among medical men in the present day is that in many instances, under the control of the reasoning and moral taculties, its use is to some extent beneficial, and therefore let those who find it so and who can strictly limit its consumption enjoy the benefit. To others, on the other hand, alcohol is a lethal weapon which works its fell effect, and therefore let those who are so affected abstain altogether from its use. It affects the weakly by reducing their energy, their vigour, their initiative, and their enterprise. It is from the indiscriminate use of alcohol by this class that disease, degeneration, crime, and brutality result. in consequence of which wise and tolerant men who have found even benefit from it in moderation have totally abstained from it and decried it in no measured terms as the most potent evil and the most active cause of sickness, disease, and death. This being so, it has been asked, "Why do men drink?" and the general answer, we are bound to confess, is, as Shadwell states, that alcohol in one form or another exhilarates, removes depression, lightens pain, and makes glad the heart. It tends to foster conviviality and good fellowship, to make life brighter and more enjoyable, and to make man pleased with himself and others.

\section{General EFFects of Alcoholism.}

As to the general effects of alcoholism-if the term be applied to the whole range of disorders caused through poisoning by alcoholic liquors-the toxic action may be described as either direct upon the individual or social, far-reaching, and indirect, affecting the efficiency of the community and causing injury to the national reputation through a loss of productive labiur, an increase of pauperism, the spread of crime, and the retardation of all improvement. Through the direct influence of

1 A paper read ibefore the Society for the Study wof Inehriety on. 